\title{
Desenvolvimento de uma Bancada Didática para Práticas de Controle de Velocidade de Motor de Corrente Contínua
}

\author{
Diego S. Torga, José A. N. Cocota Júnior, Alan K. Rêgo Segundo, \\ Wellington T. Martins \\ Escola de Minas, Universidade Federal de Ouro Preto, Departamento de \\ Engenharia de Controle e Automação, Ouro Preto, MG, Brasil (e-mail: \\ diegotorga@hotmail.com,cocota@em.ufop.edu.br, \\ alankardek@em.ufop.edu.br,wellinton-2011@live.com).
}

\begin{abstract}
The didadict bench is a tool that university professors use to provide students with an experience that allows the practical application of the theoretical content seen in class. It presents the difficulties faced in a real system, and enables the student to develop professional competence. This paper presents the development of a didactic bench for a direct current motor speed control practice. It shows its manufacture, engine layout, power and control circuit, graphical interface by MATLAB, and the use of Arduino as a control platform. In addition, it presents some students evaluation of the bench itself, who have used it.

Resumo: A bancada didática é um recurso utilizado por docentes nas universidades, para proporcionar ao aluno uma experiência, que permite a aplicação prática do conteúdo teórico ensinado em sala. Ela apresenta as dificuldades enfrentadas num sistema real, propiciando ao discente desenvolver habilidades e competências profissionais. Este trabalho apresenta o desenvolvimento de uma bancada didática para prática de controle de velocidade de um motor de corrente contínua. Nele é mostrada a sua fabricação, a disposição dos motores, o circuito de potência e de controle, a interface gráfica pelo MATLAB, e o uso do Arduino como plataforma de controle. Além disso, mostra a avaliação da própria bancada por alguns discentes que a utilizaram.
\end{abstract}

Keywords: Didactic Bench; Arduino; Graphical Interface; MATLAB; Control Plataform.

Palavras-chaves: Bancada Didática; Arduino; Interface Gráfica; MATLAB; Plataforma de Controle.

\section{INTRODUÇÃO}

O mercado de trabalho cada vez mais busca profissionais qualificados e que tenham a capacidade de solucionar os mais diversos problemas, seja de forma qualitativa ou quantitativa. Uma das habilidades que se espera de um profissional recémformado é justamente a capacidade de aplicar o conhecimento teórico na solução desses problemas. Além disso, que tenha a capacidade de analisar dados e gráficos e extrair as informações necessárias para a tomada de decisão. Portanto, para alguns cursos, especificamente para as engenharias, a melhor forma de consolidar essas habilidades é associar o conhecimento teórico à prática.

Dessa forma, a utilização de bancadas didáticas e outros recursos tecnológicos que auxiliem o processo de ensinoaprendizado - proporcionando experimentos e projetos práticos que complementem a formação de um profissional - estão se tornando comuns no ensino de graduação nas áreas da engenharia (Shui-Chun and Ching-Chih, 2009) (Medeiros et al., 2011). Essa prática tornou-se uma indispensável metodologia para motivar os alunos de graduação uma vez que "[...] o método tradicional - apresentar ao estudante não o problema, mas a solução acaba privando este estudante de todo a excitação, corta o impulso criativo e reduz a aventura da humanidade a um monte empoeirado de teoremas" (Dorf and Bishop, 2001).
No entanto, a maioria das plataformas experimentais disponíveis e aplicáveis para a graduação e pós-graduação são muito caras e/ou tem uma arquitetura de hardware e software fechada. Além disso, o uso exclusivo dessas plataformas impede a possibilidade dos alunos desenvolverem uma das características mais importantes de um profissional de engenharia, que é a capacidade de projetar, gerenciar e executar um projeto (Cocota et al., 2013).

Consequentemente, diversas plataformas têm sido desenvolvidas. Em Kardek, Cocota e Ferreira (2015), é apresentada a elaboração de uma ferramenta educacional em conjunto a um sistema supervisório para aplicações em controle de temperatura. Já em Gunasekaran e Potluri (2012), é descrito um módulo laboratorial de controle baseado em um servo motor de corrente contínua (CC) que permite aos alunos realizarem uma ampla variedade de experimentos baseados em projetos de controladores, e.g, Proporcional - Integral - Derivativo (PID), realimentação de estado e controle adaptativo. Da mesma maneira, Yime, Villa e Paez (2007), propõem o controle de motores CC para aplicações em robótica de baixo custo baseados em técnicas de controle comumente utilizados nessa área, e.g, o controle PID em cinemática inversa e o controle dinâmico por torque calculado.

Considerando os projetos supracitados, no trabalho proposto buscou-se unir duas características para o projeto da 
bancada: custo relativamente baixo e a capacidade de ser empregada como instrumento didático para contribuir na formação acadêmica e profissional dos discentes.

Desta maneira, este trabalho tem como objetivo o desenvolvimento de uma bancada didática para práticas de controle da velocidade de um motor CC. Logo, é apresentado como foi desenvolvido a bancada - desde o desenvolvimento do hardware ao supervisório. Além disso, é apresentada uma prática para o controle da velocidade de um motor CC, cujo controlador é sintonizado por meio da curva de reação.

\section{A BANCADA DIDÁTICA}

Nesta seção são apresentados os principais componentes da bancada, bem como suas funcionalidades por meio da interface gráfica do usuário.

\subsection{A Descrição da Bancada}

A estrutura da bancada é constituída por um motor CC modelo "JGA25-371 DC" - como mecanismo principal - em conjunto com um encoder, com resolução de 334 pulsos por revolução, conectado ao mesmo. É , também, possível acoplar um motor de corrente alternada (CA) de polos sombreados, alimentado em CC propositalmente para operar como um freio de modo a provocar um distúrbio ao motor CC (Fig. 1).

O circuito eletrônico foi projetado no formato de um shield - que se conecta à parte superior do Arduino - para o acionamento de ambos os motores. Ele possibilita duas formas de operação: automática e manual, de maneira que, ambas podem ser acionadas tanto por meio da interface gráfica do usuário, quanto pelo próprio hardware.

Para alimentação do circuito de potência e do motor de polos sombreados, utilizou-se uma fonte de $36 V_{c c}$ de 1 A. Já para o motor CC, foi utilizado um regulador de tensão de 12 $V_{c c}$ de $1 \mathrm{~A}$. Por fim, para a alimentação do Arduino UNO foram utilizados $5 V_{c c}$ de uma porta USB.

\subsection{O Projeto de Hardware}

A ligação da fonte externa ao hardware é realizada por meio do conector plug Jack P4. Assim, as conexões, incluindo o Arduino, dispõe de um mesmo referencial comum. Fisicamente, para tal fim, foi necessário uma barra de pinos interligando o plug P4 ao ponto GND (Figura 2 - a). Para as demais necessidades do uso de energia (led's de indicação de operação manual ou automática, encoder, potenciômetros e botão), é empregada a própria alimentação do Arduino de $5 V_{c c}$ proveniente do computador. Por meio da barra de pinos, é possível observar o ponto comum entre o computador, a fonte externa e o Arduino (Figura 2 - b). A alimentação dos motores é realizada de forma paralela, sendo o motor CA ligado diretamente à saída do plug $\mathrm{P} 4\left(36 V_{c c}\right)$ e o motor $\mathrm{CC}\left(12 V_{c c}\right)$ ligado à saída do regulador de tensão (Figura 2 - c).

Em relação ao acionamento dos motores com o Arduino (Fig. 3), foram utilizados transistores ligados a saídas PWM's do Arduino para amplificarem a corrente, assim como o emprego de diodos antiparalelos "roda livre"para a proteção do circuito. Os pontos JP01 e JP02 são para futuros trabalhos que possibilitem a estimação da velocidade para o motor $\mathrm{CC}$ e o

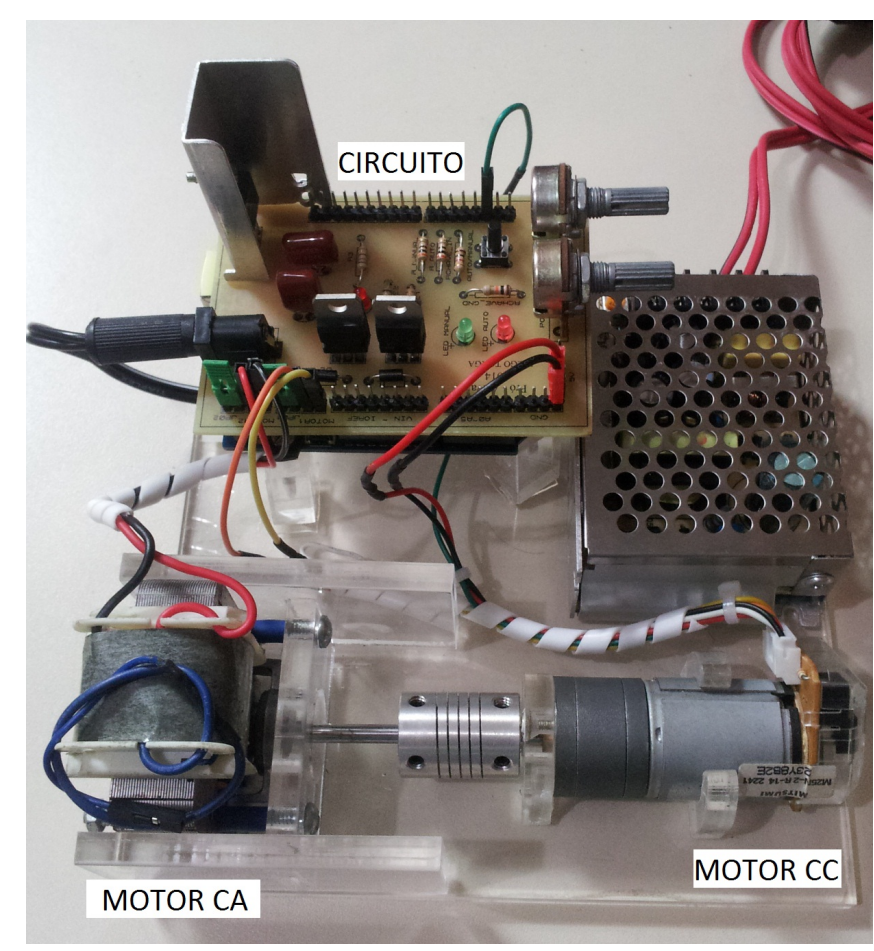

Figura 1. A Bancada do Controle de Velocidade de Motor CC.

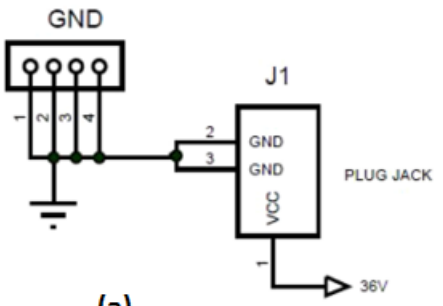

(a)

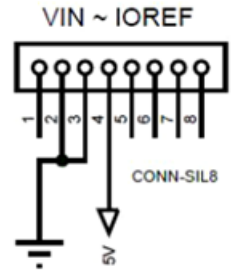

(b)

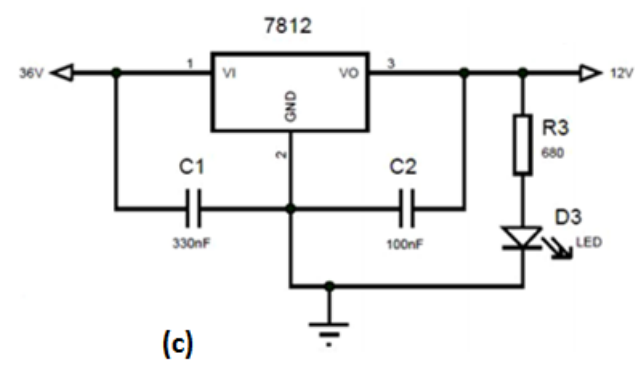

Figura 2. (a) Conexão da Fonte ao Hardware; (b) Alimentação Arduino ao Hardware ; (c) Ligação Regulador de Tensão .

distúrbio no sistema por meio da leitura da corrente por técnicas sensorless.

O status automático e manual pode ser definido tanto pelo botão presente no hardware (Figura 4 - a) quanto pelo supervisório (Fig. 10). Abaixo, temos a representação dos potenciômetros utilizados no modo manual (Figura 4 - c), no qual foram utilizadas as entradas analógicas do Arduino que possuem 8bits de resolução, posteriormente, convertidas em sinais PWM's de 10bits. Para facilitar a identificação do modo de operação foi disposto no circuito um par de led's para sinalização (Figura 4 - b).

Na Tabela 1 consta a descrição das conexões entre o hardware e o Arduino. 


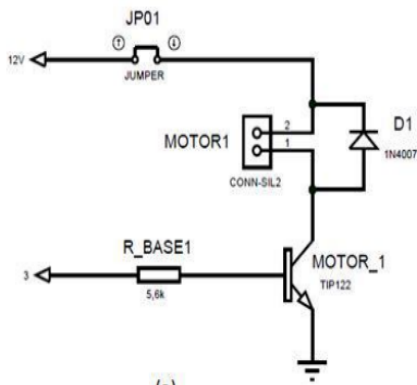

(a)

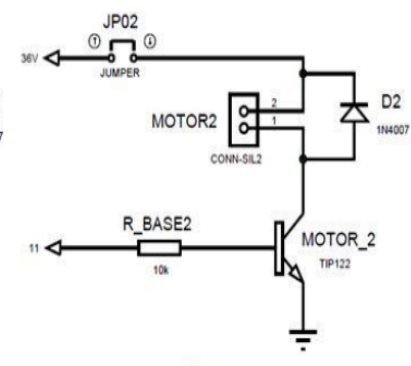

(b)
Figura 3. Acionamento Motores, (a) Motor CC, (b) Motor de Polos Sombreados.

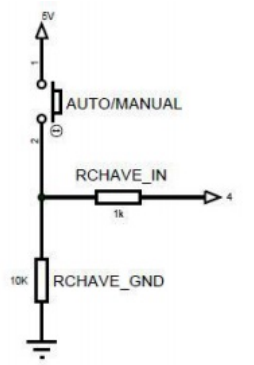

(a)

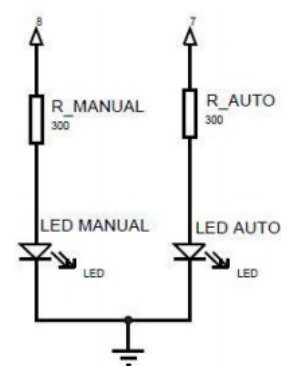

(b)

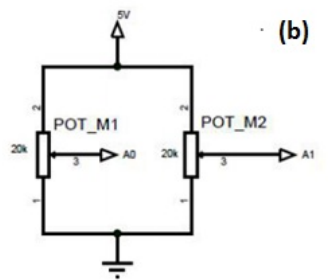

(c)

Figura 4. (a) Circuito Botão Manual/Automático; (b) Circuito Led's; (c) Potenciômetros do Modo Manual.

Tabela 1. Diagrama de Conexão do Hardware e o Arduino.

\begin{tabular}{|c|l|}
\hline \multicolumn{2}{|c|}{ Hardware e Arduino } \\
\hline Pinagem & \multicolumn{1}{c|}{ Função } \\
\hline A0 & $\begin{array}{l}\text { Leitura - potenciômetro motor CC } \\
\text { (POT_M1). }\end{array}$ \\
\hline A1 & $\begin{array}{l}\text { Leitura - potenciômetro motor } \\
\text { de polos sombreados (POT_M2). }\end{array}$ \\
\hline 3 & $\begin{array}{l}\text { Sinal do PWM para o motor CC } \\
\text { (R_BASE1). }\end{array}$ \\
\hline 7 & Leitura - botão (RCHAVE_IN). \\
\hline 7 & $\begin{array}{l}\text { Acionamento do led manual - } \\
\text { verde (R_MANUAL). }\end{array}$ \\
\hline 11 & $\begin{array}{l}\text { Acionamento do led } \text { automático - } \\
\text { vermelho (R_AUTO). }\end{array}$ \\
\hline $5 V_{c c}$ & $\begin{array}{l}\text { Sinal do PWM para o motor de } \\
\text { polos sombreados (R_BASE2). }\end{array}$ \\
\hline $\begin{array}{l}\text { Alimentação dos circuitos de 5 } V_{c c} \text { e } \\
\text { da barra de pinos de 5 } V_{c c} \text { para } \\
\text { as demais necessidades. }\end{array}$ \\
\hline GND & $\begin{array}{l}\text { Aterramento da placa e da barra } \\
\text { de pinos GND para as demais } \\
\text { necessidades. }\end{array}$ \\
\hline
\end{tabular}

\subsection{Interface Gráfica do Usuário}

A interface com o usuário permite realizar, experimentalmente, a identificação do sistema, projetar o controlador e rea- lizar ensaios de operação do sistema em malha aberta e malha fechada (Fig. 5).

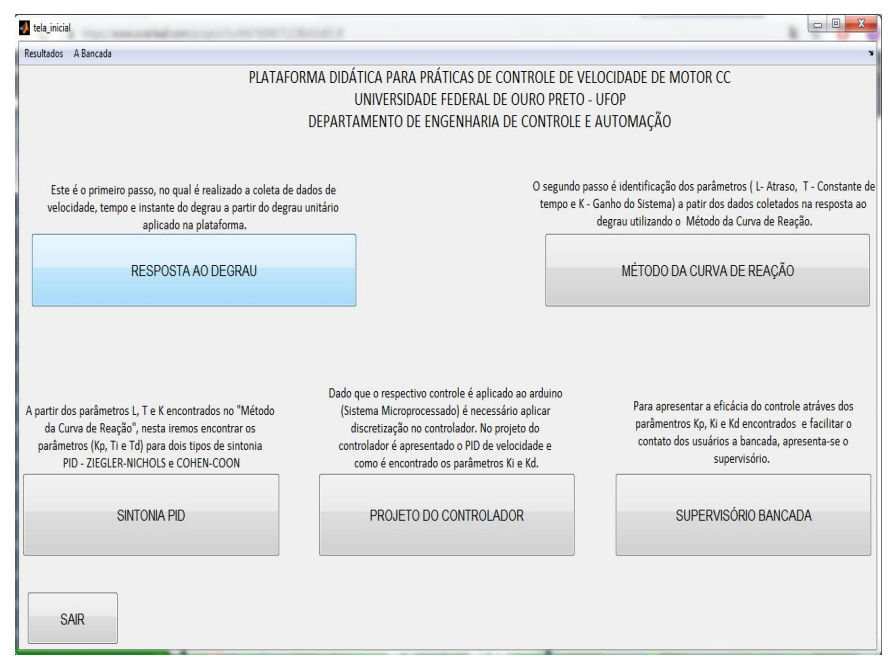

Figura 5. Tela Inicial da Interface Gráfica.

O primeiro botão é relacionado ao experimento da resposta ao degrau (Fig. 6). Nessa tela o usuário configura a comunicação com o Arduino, o ajuste do valor da tensão inicial na entrada do motor CC, o percentual de acréscimo no fundo de escala desse valor para que o degrau seja efetuado, bem como o intervalo de amostragem para $8 \mathrm{~ms}$ ou $10 \mathrm{~ms}$. Os dados de velocidade (RPM) e tempo (ms) nesse experimento são armazenados no Arduino e enviados ao término do experimento para o MATLAB por meio da porta USB.

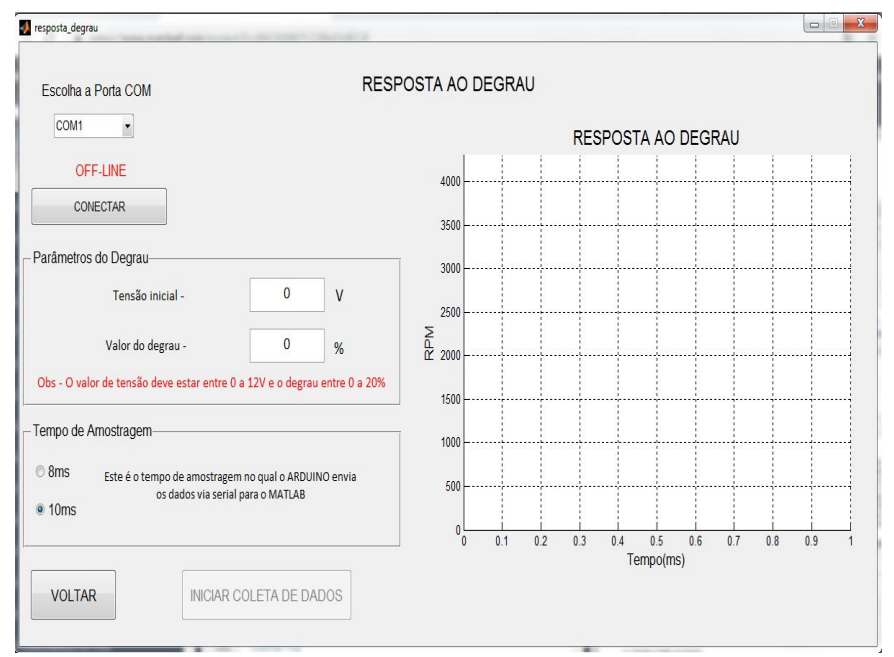

Figura 6. Tela Resposta ao Degrau.

Em sequência, após realizado o ensaio da resposta ao degrau, a próxima etapa consiste em encontrar os parâmetros ganho direto $(\mathrm{K})$, constante de tempo $(\mathrm{T})$ e o atraso (L) com o auxílio da tela Método da Curva de Reação (Fig. 7). Assim, com o modelo de primeira ordem com atraso que foi identificado a partir do experimento da curva de reação, é determinado os parâmetros do controlador PID para as sintonias de ZieglerNichols e Cohen-Coon (Fig. 8). Uma vez obtido esses parâmetros, o PID é discretizado conforme a equação presente na tela Projeto do Controlador Digital (Fig. 9), considerando o intervalo de amostragem $(\tau)$ de $5 \mathrm{~ms}$. O algoritmo de controle é implementado no firmware do Arduino. 


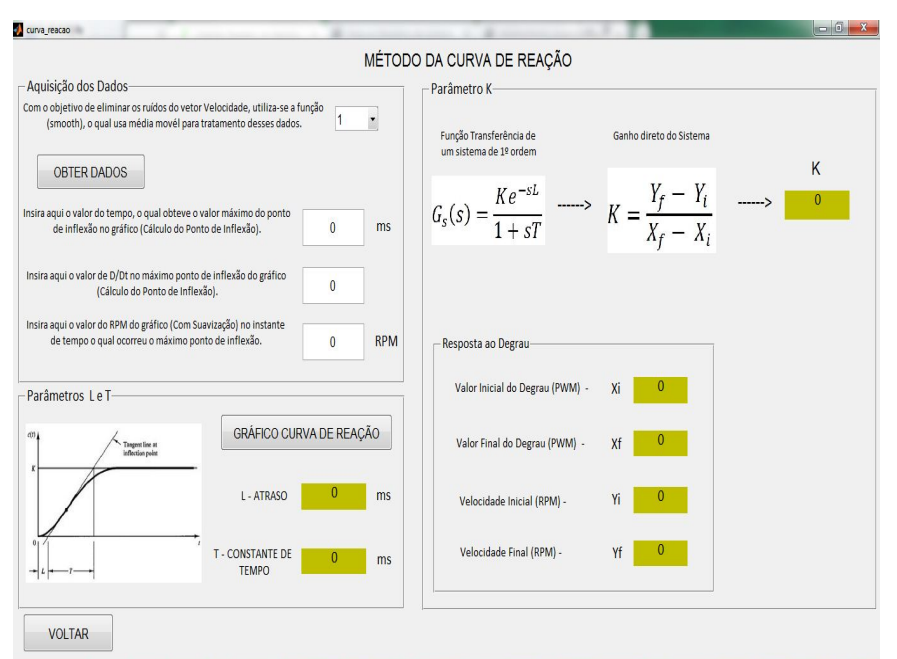

Figura 7. Tela Método da Curva de Reação.

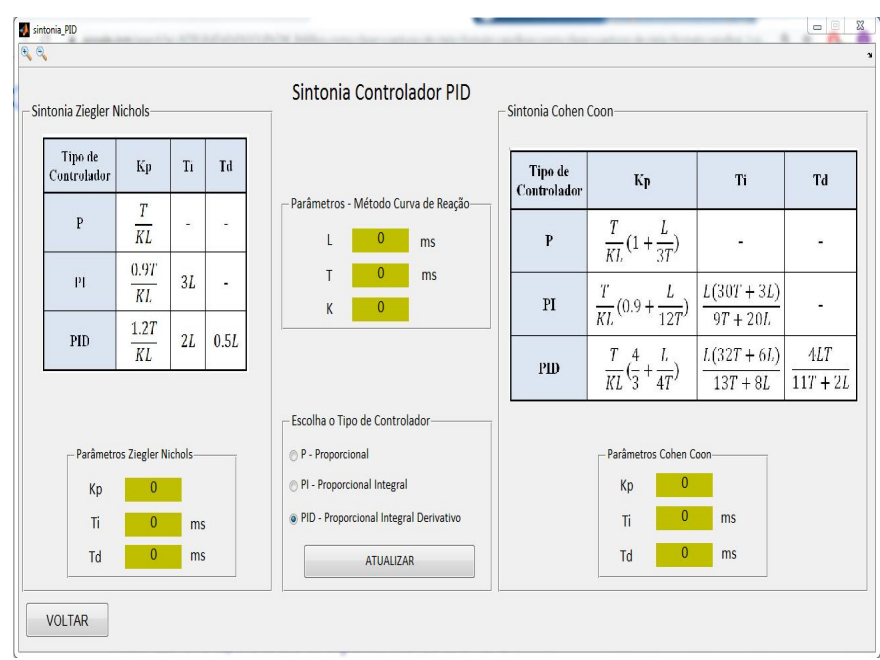

Figura 8. Tela Sintonia do Controlador PID.

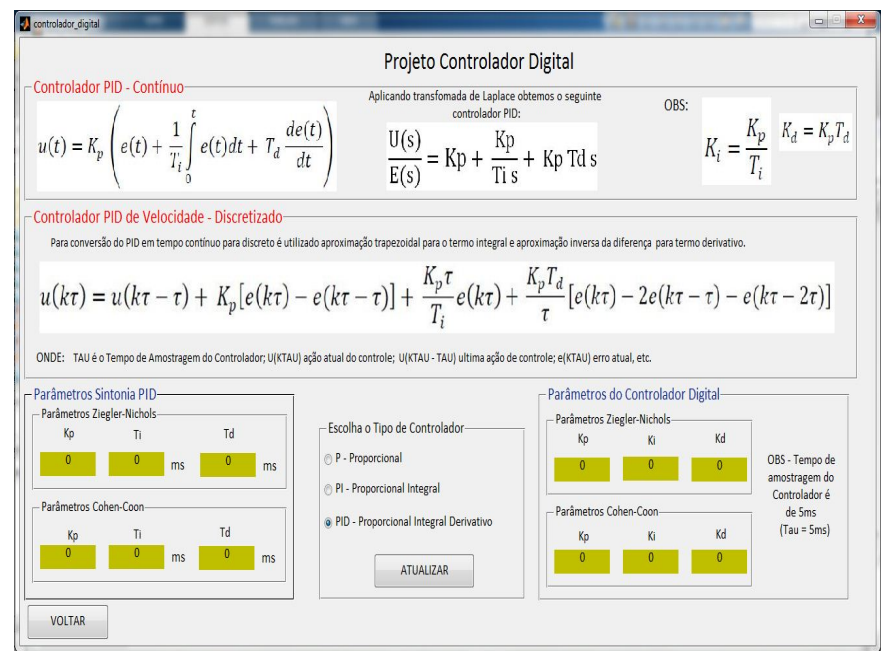

Figura 9. Tela Projeto do Controlador Digital.

Finalmente, é apresentada a tela Supervisório (Fig. 10), no qual é possível visualizar o controle de velocidade da bancada do motor CC com a exibição de informações do processo, e.g, velocidade do motor CC (sinal de saída), tipo de operação (Manual/Automático), o valor de referência (Set Point), o sinal de entrada, o erro, os parâmetros do controlador e o estado de acionamento (ligado/desligado) do motor de polos sombreados. Os parâmetros do controlador podem ser atualizados pelo usuário, não sendo limitado aos valores sintonizados por meio da curva de reação. As variáveis que permitem a análise do experimento (velocidade, valor de referência, erro e o sinal de controle) podem ser visualizadas e salvas pelo usuário para posterior estudo. No modo de operação de controle, os dados que são exibidos na tela Supervisório são atualizados a cada $100 \mathrm{~ms}$.

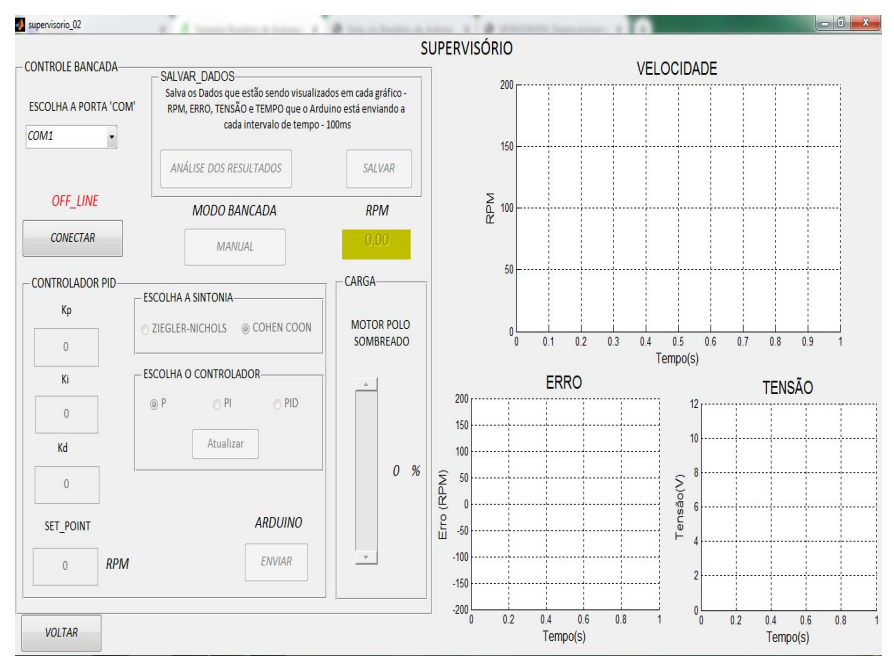

Figura 10. Tela do Supervisório.

\section{O FUNCIONAMENTO DA BANCADA}

Nesta seção é apresentado os resultados experimentais do sistema em malha fechada para ilustrar a aplicabilidade da bancada nas disciplinas de controle. Os parâmetros do controlador PI (Tabela 2) utilizados nos experimentos foram obtidos por meio da curva de reação pelos métodos de sintonia por Ziegler Nichols e Cohen Coon para $\mathrm{L}=8.6 \mathrm{~ms}$ e $\mathrm{T}=2.6 \mathrm{~ms}$.

Tabela 2. Ganhos do Controlador PI

\begin{tabular}{lll}
\hline Ganhos & Ziegler Nichols & Cohen Coon \\
\hline$K_{p}$ & 0.017 & 0.022 \\
$K_{i}$ & 25.934 & 4.614
\end{tabular}

\subsection{Resposta do Controlador sem Alteração de Carga no Motor}

Considerou a bancada sem o acoplamento de motor de polos sombreados. Para essa configuração realizou-se o experimento de controle de velocidade do motor $\mathrm{CC}$ de acordo com as referências de 140 RPM, 90 RPM, 115 RPM e 100 RPM para o controlador obtido mediante as sintonizações de Ziegler Nichols (Fig. 11) e Cohen Coon (Fig. 12).

Em ambos os experimentos para o intervalo de dados apresentado, o sistema estava em malha fechada. Nota-se que o tempo de acomodação do sistema para o controlador Cohen Coon é inferior ao de Ziegler Nichols. Isso reforça o conceito de que o controlador obtido por meio de Cohen Coon apresenta uma menor dependência dos valores da constante de tempo (T) e do atraso (L) em relação ao controlador obtido por meio de Ziegler Nichols. 


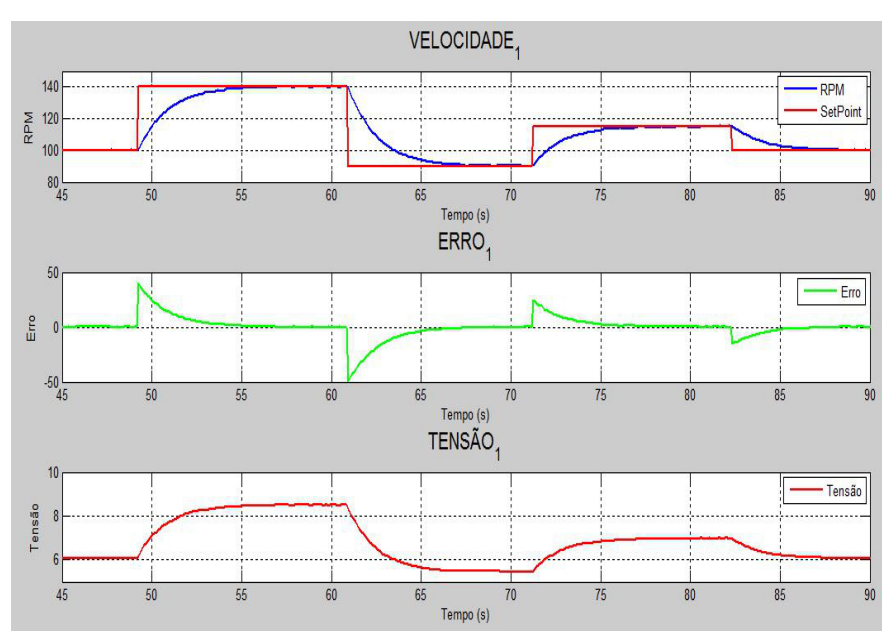

Figura 11. Resposta do Controlador PI Sintonizado por Ziegler Nichols.

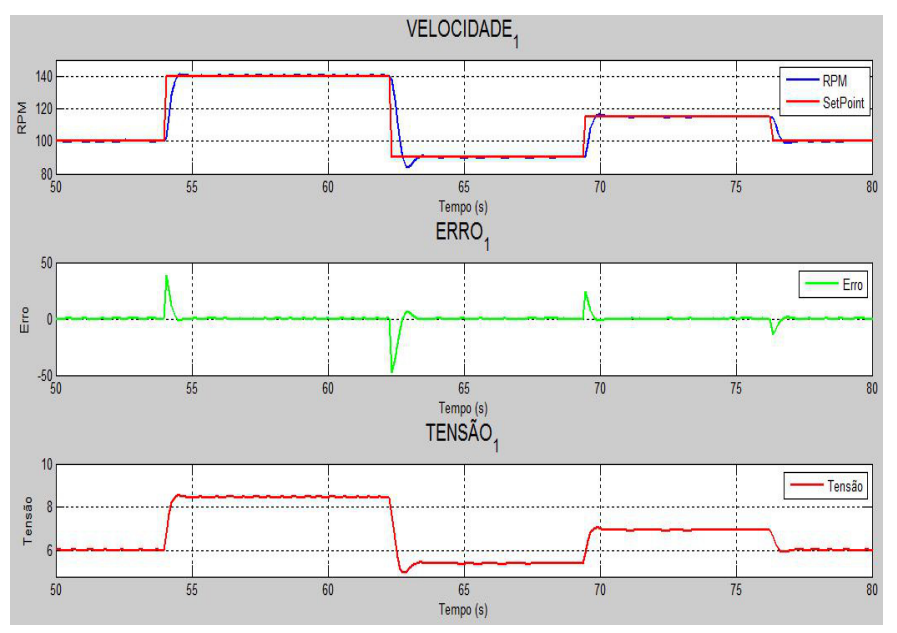

Figura 12. Resposta do Controlador PI Sintonizado Cohen Coon.

\subsection{Resposta do Controlador com Alteração de Carga no Motor}

Na segunda configuração do experimento, o eixo do motor de polos sombreados foi acoplado ao eixo do motor CC para ser acionado em determinados períodos, com o intuito de gerar distúrbios no sistema. Utilizou-se o controlador sintonizado pelo método Cohen Coon para as referências de 100 RPM e 115 RPM (Fig. 13).

Para a análise, o experimento foi dividido em três intervalos de tempo: (1) tendo em consideração o sistema em malha aberta, no ponto de operação de 100 RPM, o motor de polos sombreados foi acionado por um período de tempo; no qual resultou em um erro em regime permanente no período de operação como uma carga adicional ao processo; na figura 13 não há registro desse erro, uma vez que o sistema estava em malha aberta e o mesmo não foi computado; (2) para o sistema em malha fechada no ponto de operação de 115 RPM, acionouse o motor de polos sombreados, cujo o erro resultante desse distúrbio foi compensado pelo controlador; (3) Por fim, o motor de polos sombreados é desenergizado resultando na aceleração do sistema em função da retirada da carga que é compensada pelo controlador.

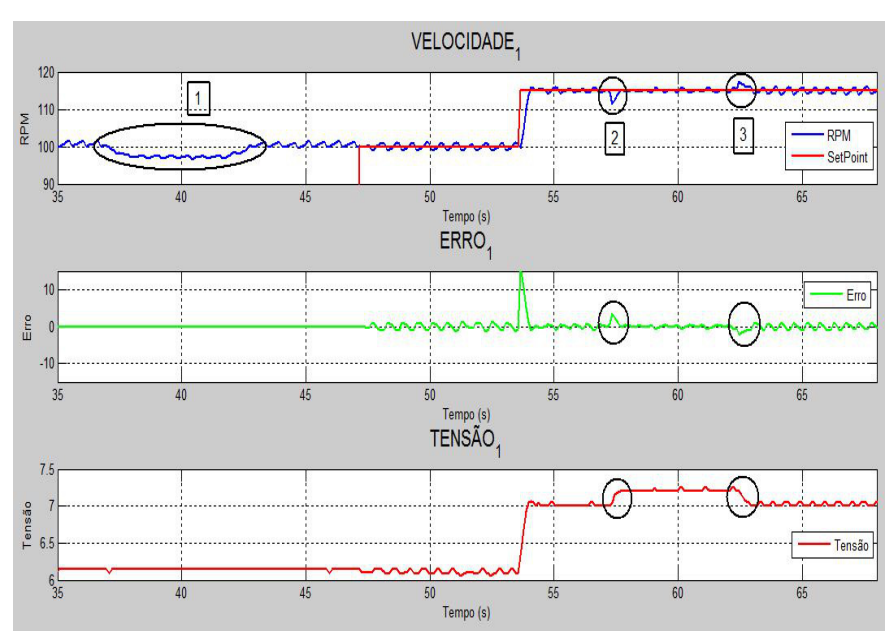

Figura 13. Resposta do Controlador PI para L $=8.6446 \mathrm{~ms}$ e $\tau=2.6318 \mathrm{~ms}$ Sintonia Cohen Coon com Alterações na Carga.

\section{RESULTADOS}

O principal resultado foi o desenvolvimento de uma bancada com baixo custo de hardware (aproximadamente $\mathrm{R} \$$ 200,00), que possibilita assistir a formação acadêmica dos discentes de Engenharia na área de Controle, por meio de experimentos para identificação de sistemas, projeto de controladores, tal como o estudo de desempenho do sistema em malha fechada. Para analisar a contribuição do uso desta ferramenta em disciplinas de Teoria de Controle, um questionário foi aplicado aos estudantes que utilizaram a bancada em práticas no período de 2018.2 e 2019.1. Ao todo, 22 alunos responderam de forma voluntária o questionário. De acordo com os alunos, a bancada possibilitou visualizar conceitos de engenharia de controle $(95,45 \%)$, contribuiu no melhor entendimento de um controlador $(95,45 \%)$, bem como motivou no estudo da disciplina $(90,90 \%)$. Na opinião da maioria dos discentes o nível de interatividade da bancada foi adequado $(86,37 \%)$, sendo de fácil uso e entendimento (77,27\%). Alguns alunos registraram no formulário a importância de que projetos como esse sejam desenvolvidos na universidade para contribuir em uma melhor capacitação dos discentes do curso de graduação em Engenharia de Controle e Automação.

Tabela 3. Avaliação dos Alunos - A = Concordo Plenamente, $\mathrm{B}=$ Concordo, $\mathrm{C}=$ Neutro, $\mathrm{D}=$ Discordo e $\mathrm{E}=$ Discordo Totalmente

\begin{tabular}{|l|l|l|l|l|l|}
\hline \multicolumn{1}{|c|}{ Respostas } & A\% & B\% & C\% & D\% & E\% \\
\hline $\begin{array}{l}\text { 1. A bancada me ajudou a } \\
\text { visualizar os conceitos de En - } \\
\text { genharia de Controle. }\end{array}$ & 77,27 & 18,18 & 4,55 & 0 & 0 \\
\hline $\begin{array}{l}\text { 2. Consegui desenvolver um } \\
\text { melhor entendimento de co - } \\
\text { mo controlar a velocidade de } \\
\text { um motor de CC. }\end{array}$ & 54,54 & 40,91 & 4,55 & 0 & 0 \\
\hline $\begin{array}{l}\text { 3. A execução da prática me } \\
\text { motivou no estudo da disci - } \\
\text { plina. }\end{array}$ & 68,18 & 22,72 & 9,10 & 0 & 0 \\
\hline $\begin{array}{l}\text { 4. O nível de interatividade } \\
\text { da bancada foi adequado. }\end{array}$ & 59,10 & 27,27 & 13,63 & 0 & 0 \\
\hline $\begin{array}{l}\text { 5. A bancada foi fácil de usar } \\
\text { e entender. }\end{array}$ & 40,91 & 36,36 & 13,63 & 9,10 & 0 \\
\hline $\begin{array}{l}\text { 6. O período para a execução } \\
\text { das práticas foi adequado. }\end{array}$ & 40,91 & 40,91 & 13,63 & 4,55 & 0 \\
\hline
\end{tabular}




\section{CONCLUSÕES E TRABALHOS FUTUROS}

Este trabalho abordou o desenvolvimento de uma bancada didática de baixo custo para práticas de controle da velocidade de um motor de corrente contínua. O custo do software MATLAB não foi considerado, uma vez que depende do número de licenças. A plataforma desenvolvida possibilita explorar conceitos de identificação de sistemas, projeto de controladores, assim como a análise de sistemas em malha fechada quando sujeitos a distúrbios, configurando um sistema didático com grande potencial para uso em disciplinas de Engenharia de Controle. Em trabalhos futuros, deseja-se: (1) disponibilizar a bancada para acesso remoto, ampliando sua disponibilidade para práticas remotas; (2) utilizar técnicas de sensorless para estimar a velocidade do motor CC e o de polos sombreados por meio da amostragem da corrente nos motores.

\section{REFERÊNCIAS}

Cocota, J.A.N.J., Abrão, D.C., Lopes, A.G., Medeiros, M.R.O., and Lopes, E.S.S. (2013). Development of tangible experiments for motivating undergraduate students. IEEE Global Engineering Education Conference (EDUCON), 497-506. Berlin: [s.n.].

Dorf, R.C. and Bishop, R.H. (2001). Sistemas de Controle Modernos. $8^{\mathrm{a}}$. ed. Rio de Janeiro: LTC. ISBN 0-201-308649.

Gunasekaran, M. and Potluri, R. (2012). Low-cost undergraduate control systems experiments using microcontroller-based control of a dc motor. IEEE Transactions on Education, 55(4), 508 - 516. Blumenau: [s.n.].

Medeiros, R.L.P., Araújo, I.L.C., de Freitas, V.S., and Silva, O.F. (2011). Desenvolvimento e aplicação de motores de corrente contínua virtuais aplicados nas aulas laboratoriais de controle de sistemas. XXXIX congresso brasileiro de educação de engenharia, 1 - 10. Blumenau: [s.n.].

Segundo, A.K.R., Junior, J.A.N.C., and Ferreira, D.V.M. (2015). Development of an educational tool for control engineering. IEEE Global Engineering Education Conference (EDUCON), 1 - 8. Tallinn, Estonia: [s.n].

Shui-Chun, L. and Ching-Chih, T. (2009). Development of a self-balancing human transportation vehicle for the teaching of feedback control. IEEE Transactions on Education, 1(52), 157-168.

Yime, E., Villa, J.L., and Paez, J. (2014). Design of a brushed dc motors pid controller for development of low-cost robotic applications. 2014 III International Congress of Engineering Mechatronics and Automation (CIIMA), 1 - 4. Cartagena, Colombia: [s.n.]. 